\begin{tabular}{|c|c|}
\hline Title & Switching mechanism of sensor-motor coordination through an oscillator network model \\
\hline Author(s) & Funato, Tetsuro; Kurabay ashi, Dai suke; Nara, Masahito; A onuma, Hitoshi \\
\hline Citation & $\begin{array}{l}\text { IEEE Transactions on Systems Man and Cybernetics Part B (Cybernetics), 38(3), } 764.770 \\
\text { https://doi.org/10.1109/ SMCB.2008.922060 }\end{array}$ \\
\hline Issue Date & $2008-06$ \\
\hline Doc URL & http:/hdl.handle.net/2115/34766 \\
\hline Rights & $\begin{array}{l}\text { O2008 IEEE. Personal use of this material is permitted. However, permission to reprint/republish this material for } \\
\text { advertising or promotional purposes or for creating new collective works for resale or redistribution to servers or lists, } \\
\text { or to reuse any copyrighted component of this work in other works must be obtained from the IEEE. }\end{array}$ \\
\hline Type & article \\
\hline File Information & getPDF.pdf \\
\hline
\end{tabular}

Instructions for use 


\title{
Switching Mechanism of Sensor-Motor Coordination Through an Oscillator Network Model
}

\author{
Tetsuro Funato, Student Member, IEEE, Daisuke Kurabayashi, Member, IEEE, Masahito Nara, and Hitoshi Aonuma
}

\begin{abstract}
Insects have small brains, but their behavior is highly adaptive; this leads us to conclude that their brains possess a simple adaptation mechanism. This paper focuses on the pheromone processing of crickets, varying their aggression depending on their global neural connection, and proposes a behavior selection mechanism that can be controlled by network transformation. The controller is composed of an oscillator network, and its behavior is decided by the synchrony of organic oscillations. Furthermore, every network component corresponds to a certain brain module. A model is realized by using an analog circuit, and it is applied to a simple robot that displays the behavior of a real insect.
\end{abstract}

Index Terms-Brain modeling, networks, nonlinear oscillators.

\section{INTRODUCTION}

$\mathbf{C}$ URRENT very large scale integration technology has made it possible to fabricate over one billion cells on a chip. The enormity of this quantity can be recognized by comparing it with the number of neurons in insect brains, which has been found to be only $10^{4}-10^{6}$. This fact has encouraged us to visualize the construction of an artificial brain system using large-scale integration. However, a methodology for organic adaptability is not available, and therefore, further research that goes beyond the discussion of cell numbers is required.

Recent research has reported multisystems (or networks) that possess various structures for different functionalities; for example, actual phenomena such as the Internet and traffic networks [1] and disease infection [2], [3] use a small-world (SW) structure to realize effective communication. Thus, the transformation of a network causes a transition in the system functionality. In particular, if the structural change is due to the environment, proper transformation implies adaptation. The use of structural changes as a medium of adaptability has not been emphasized; however, it has the potential to realize adaptability in simple systems that are modeled similar to insect brains. In fact, there are several reports of unconventional structures in animal brains, for instance, from a functional viewpoint, the presence of an SW structure in the antennal lobe has been suggested [4]. An investigation of the neural connections in nematodes led to the inference of the SW structure [5].

Manuscript received November 7, 2007. This work was supported in part by the Japanese Ministry of Education, Culture, Sports, Science, and Technology, Japan, under Grant-in-Aid 17075007 and Grant-in-Aid 17075001 for Scientific Research on Priority Areas. This paper was recommended by Associate Editor W. Dixon.

T. Funato, D. Kurabayashi, and M. Nara are with the Department of Mechanical and Control Engineering, Tokyo Institute of Technology, Tokyo 152-8552, Japan (e-mail: funato@ irs.ctrl.titech.ac.jp; dkura@irs.ctrl.titech.ac.jp; m-nara@ irs.ctrl.titech.ac.jp).

H. Aonuma is with the Research Institute for Electronic Science, Hokkaido University, Sapporo 060-0812, Japan (e-mail: aon@ncp8.es.hokudai.ac.jp).

Digital Object Identifier 10.1109/TSMCB.2008.922060
Therefore, we focus on the role of the physical structure in adaptability and aim to elucidate the brain mechanism by using a network structure.

In this paper, the olfactory system in the insect brain, particularly the unit that processes pheromones in crickets, is considered. A pheromone is a chemical substance that mediates communication among animals belonging to the same species. As the behavior caused by pheromones are stereotypical, a simple processing unit can replicate their behavior. A well-known behavioral trait of crickets is that male crickets show aggression toward or avoid other male crickets. Current researches indicate that the selection of either of the two behavioral patterns is related to global connectivity in the neural network (NN), which is based on experiences from previous fights [6], [7]; such a selection mechanism implies the existence of a structural effect. We model the processing unit using the structural characteristics of an oscillator network. A previous study has proposed a method for controlling network behavior by manipulating the structure [8], [9]. An oscillator network is capable of modeling an organic selector. Oscillations of $15-30 \mathrm{~Hz}$ are observed in olfactory units, which are the main pheromone processors of various organisms such as locusts, cockroaches, bees, and wasps [10]. Furthermore, the odor dependence of the synchrony of the oscillators is also observed [11]. This fact implies that the oscillator network model can be adapted as an actual brain model.

We develop the model of an antennal lobe by using an oscillator network that changes behavior according to its structure. We realize the model by employing an analog circuit that comprises coupled van der Pol (VDP) oscillators. Subsequently, we propose a robot that autonomously selects its behavior.

\section{Behavioral Patterns And Structure of Model}

\section{A. Pheromone Information}

When a male cricket encounters conspecific male, the two crickets become aggressive and start fighting [12]. This behavior is stereotypical, and therefore, we can associate it with a certain stimulus or condition. Further, the previous experiences of organisms affect their actions. For example, crickets avoid their previous dominants instead of attacking them at first contact [12]. However, they resume aggressive behavior after flying [13]. These phenomena indicate the existence of switching mechanisms inside the brain of the cricket, and these organic switches work as a generator for experience-based adaptability.

The fighting behavior starts with pheromone stimuli. Pheromones are detected by receptors on the surface of the antennas, and the information is transferred to the antennal lobe. From an anatomical point of view, the antennal lobe is 


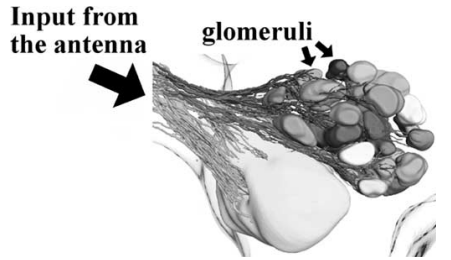

Fig. 1. Structure of antennal lobe.

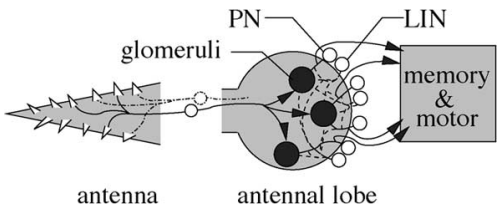

Fig. 2. Configuration of pheromone processor.

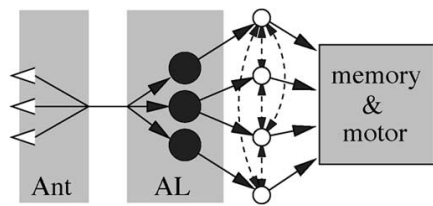

Fig. 3. Pheromone processor network.

composed of several regions, as shown in Fig. 1. The biggest part on the lower left side of the antennal lobe deals with the physical stimulus of the lobe, and the sphere-shaped parts, which are called glomeruli, on the upper right side are concerned with the processing of the chemical information, which is represented by odor. The information recorded in the antennal lobe is classified according to the type of stimulus; it is then transferred to the mushroom body via projection neurons (PNs) and subsequently is translated into a behavior [14]. Moreover, each PN is connected to local interneurons (LINs).

Fig. 2 shows the module structure in the brain involved with the flow of odor information. The arrows in the figure show the direction of information flow, whereas no arrows are drawn for the LINs because there are numerous LINs in both directions. For simplicity, we do not distinguish between excited and inhibited junctions in this paper. By considering only the connections shown in Fig. 2, we create a network model (Fig. 3) and develop a model of behavior selector using this network.

The neurons in the antennal lobe are not physically connected; however, there are gaps between the neurons, which are termed as synaptic clefts, where the information is transmitted via neurotransmitters such as gamma-aminobutyric acid and glutamate. The experience from previous fights affects this connection. Recently, researchers have reported that nitric oxide (NO) produced in the brain of the cricket during fights [6] determines the action of the insect [7], [15]. NO is produced by the neurons and expands to a large area in the brain [6], causing changes in the global synapse connections. Thus, the neural network structure translates the experience into a behavior.

\section{B. Oscillator}

Recently, the functionality of neural oscillators has attracted considerable attention, as the functionality extends from mechanical oscillators such as the central pattern generator to

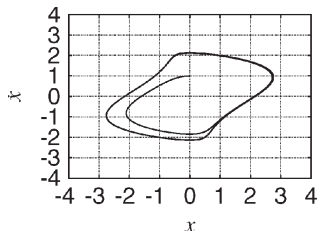

(a)

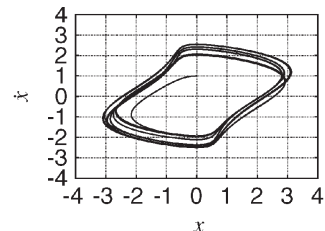

(b)
Fig. 4. Converged states of oscillators. (a) Limit cycle. (b) Quasi-periodic.

circadian rhythms, associative memory [16], and the edge detection of images [17]. Neural oscillation is also observed in the olfactory processing system of various animals [10], in which synchronous oscillation expectedly occurs for expressing odor information [11]; however, the mechanism is still under debate. The synchrony between coupled oscillators is reported to be influenced by the structure of the network [18], [19], which also affects the behavior of crickets, as mentioned in the previous section. Therefore, the functionality of the olfactory unit may be generated from its structure. In this paper, we consider the geometrical features of an oscillator network and its application to an antennal lobe model.

Some nonlinear oscillators show a stable cycle, which is termed as a limit cycle, in their phase orbit [Fig. 4(a)]. In coupled oscillators, this cycle is usually observed when they are synchronized. On the other hand, if the oscillators are not synchronized, the behavior becomes totally different [Fig. 4(b)]. As an example, we consider the VDP oscillator, which is a wellknown limit-cycle oscillator, given by

$$
\ddot{x}_{i}-\epsilon_{i}\left(1-x_{i}^{2}\right) \dot{x_{i}}+\omega_{i}^{2} x_{i}=0
$$

and we assume a linear interaction as follows:

$$
x_{i}(t+1)=\tilde{x}_{i}(t)+\lambda\left\{\frac{1}{N_{i}(t)} \sum_{j=1}^{N_{i}(t)} x_{j}(t)-x_{i}(t)\right\} .
$$

Here, $\tilde{x}_{i}(t)$ denotes an oscillator state that proceeds without interaction [only by (1)], $N_{i}$ is the number of oscillators connected to the $i$ th oscillator, and $\lambda$ is the connection coefficient. Two oscillators, whose eigenfrequencies are 1.0 and 0.9 and which are connected with $\lambda=0.01$, show a stable cycle by synchronization, as shown in Fig. 4(a). On the other hand, an oscillator system with eigenfrequencies that are 1.0 and 0.7 does not have a stable cycle [Fig. 4(b)]. This shows that the synchrony between coupled oscillators critically depends on the eigenfrequencies. Therefore, we can control the behavior of the oscillators by selecting a proper pair with regard to the eigenfrequencies.

Next, we consider the effect of the structure of the connection on coupled oscillator systems. In Fig. 5(a), the oscillators represented by solid circles have forces acting on them from the two neighboring oscillators, which determine the synchrony. If we add a new connection (we hereafter refer to it as a "shortcut") to this system, which is as shown in Fig. 5(b), the oscillator represented by the solid circle is also influenced by its new neighbor. If the eigenfrequency of the new oscillator is significantly different from the synchronized frequency, the state of synchronization will get disrupted. On the other hand, if the eigenfrequency of the new oscillator is between that of 


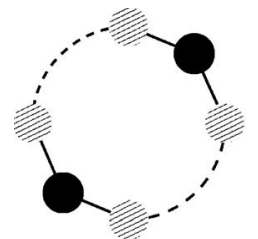

(a)

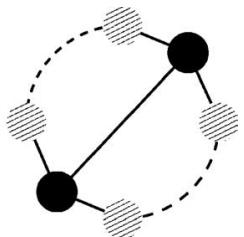

(b)
Fig. 5. Two types of connections. (a) No shortcut. (b) With shortcut.

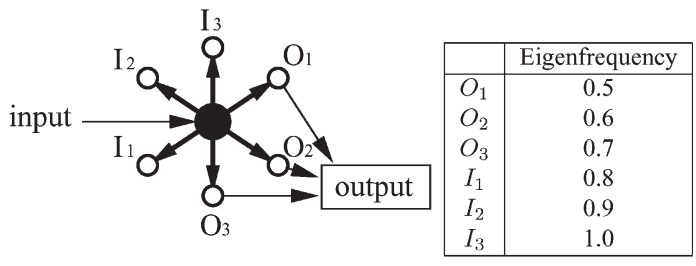

Fig. 6. No shortcuts.

the existing coupled oscillators, the two asynchronized systems will become synchronized. The procedure to control the stability of the oscillator network is more precisely studied on coupled VDP oscillators [8] and the network of the Kuramoto model [9], which results in the derivation of the threshold value of the eigenfrequency that disrupts the synchrony of the oscillators. The stability of synchronization has also been discussed without the explicit use of eigenfrequencies, which is by considering the eigenvalues of the connectivity matrix. The application of the master stability function [20] to various network types [18], [21], particularly by Jadbabaie et al. [19], has led to the development of a synchronization condition for the Kuramoto model. However, the effect of the eigenfrequencies cannot be suppressed for retaining the possibility of achieving an asynchronized state [19], and thus, the effect of the eigenvalues is comparatively suppressed when the number of network nodes is low. The number of nodes in an objective network, i.e., the number of biological organs modeled by the oscillator (glomerulus, which is described in the following section), is small (less than 1000 [10]); therefore, in this paper, we stress on the effect of eigenfrequencies instead of eigenvalues.

Next, we model a neural connection using oscillator coupling, LINs as shortcuts, and the system behavior as the basis of the synchrony of each oscillator. As a result, by setting an appropriate eigenfrequency of the oscillator, the system behavior changes with the network transformation.

\section{Functional Model Using Oscillator Network}

In this section we consider a model of olfactory unit functions based on biological knowledge (Section II-A) and the oscillator network (Section II-B).

1) Odor Classification: First, we construct a function to classify the odor information by the synchrony of the oscillators. By focusing our attention on the information flowing through a glomerulus (shown in Fig. 3), an oscillator network model is constructed as shown in Fig. 6; in this model, the information is processed through several oscillators to provide the output behavior. The circles in the figure represent oscillators, and the arrows indicate the orientation of the influence. Each oscillator has a different eigenfrequency, and therefore,
TABLE I

INPUT-OUTPUT RELATIONSHIP

\begin{tabular}{|c|c|}
\hline Input frequency & Synchronised oscillators \\
\hline 0.45 & nothing \\
0.55 & $\mathrm{O}_{1}, \mathrm{O}_{2}$ \\
0.65 & $\mathrm{O}_{2}, \mathrm{O}_{3}$ \\
\hline
\end{tabular}

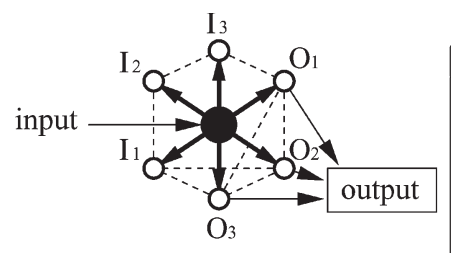

\begin{tabular}{|c|c|}
\hline & Eigenfrequency \\
\hline$O_{1}$ & $(0.5 \rightarrow) 0.65$ \\
$O_{2}$ & $(0.6 \rightarrow) 0.65$ \\
$O_{3}$ & $(0.7 \rightarrow) 0.65$ \\
$I_{1}$ & $(0.8 \rightarrow) 0.65$ \\
$I_{2}$ & 0.9 \\
$I_{3}$ & 1.0 \\
\hline
\end{tabular}

Fig. 7. With shortcuts.

TABLE II

IN-OUT RELATIONSHIP AND CORRESPONDING BEHAVIORS

\begin{tabular}{|c|cc|c|}
\hline Input freq. & \multicolumn{2}{|c|}{ Synchronised oscillators } & Behaviour \\
\hline 0.45 & (nothing $\rightarrow)$ & nothing & (neglect $\rightarrow$ ) neglect \\
0.55 & $\left(O_{1}, O_{2} \rightarrow\right)$ & nothing & (fight $\rightarrow$ ) neglect \\
0.65 & $\left(O_{2}, O_{3} \rightarrow\right)$ & $O_{1}, O_{2}, O_{3}$ & $($ fight $\rightarrow$ ) avoidance \\
\hline
\end{tabular}

synchronization does not occur without stimuli. When stimulus is provided, the glomerulus (the center circle) starts oscillating according to the signal type, and the oscillation is transferred to other oscillators; thus, synchronization occurs. We investigate the behavior of an oscillator network with given eigenfrequencies. The oscillators are expected to synchronize when the gap between the eigenfrequencies of neighboring oscillators is below 0.05 . We consider a six-oscillator system, whose eigenfrequencies range from 0.5 to 1.0 (Fig. 6). By stimulating the center device so that it oscillates with a frequency from 0.45 to 0.65 , the synchronization relationship varies, as shown in Table I. Therefore, we can observe the alternation of synchronization according to the input stimuli.

2) Behavior Switching: Next, the mechanism of behavior switching is considered. As mentioned previously, NO is produced in the antennal lobe during a fight and changes the junctions of the LINs to cause a change in attitude. In the proposed model, this function is realized by altering the connections between the oscillators.

We consider a network (see Fig. 7) whose structure is changed by new shortcuts. In this case, oscillators $O_{1}, O_{2}, O_{3}$, and $I_{1}$ are strongly connected to each other, and therefore, we can assume that these oscillators oscillate at a frequency of 0.65 on synchronization. Furthermore, if we stimulate this system using the same inputs as those in the odor classification study discussed previously, the synchronized relationships can be described as given in Table II. This result shows that behavior changes with structure, even for identical signals; therefore, the model represents the $\mathrm{NN}$ of crickets in which the behavior is controlled by NO. Thus, we have developed a brain model that adopts a behavioral pattern based on experience.

\section{Realization of Model Using Electric Circuit}

One of the primary motivations of this paper is the difference in the number of components between insect brains and computer systems, and the derivation of simple mechanisms for achieving certain functions is the final aim. For achieving this simplicity in the sense of the number of elements, the model 


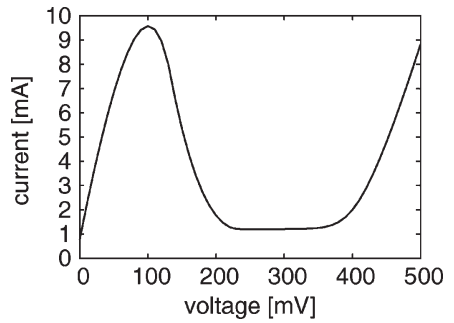

Fig. 8. $\quad V-I$ characteristics of tunnel diode (1N3858).

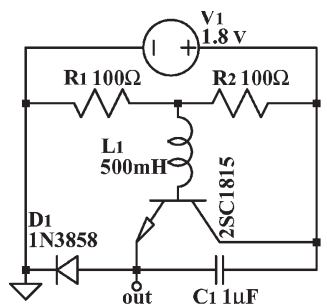

(a)

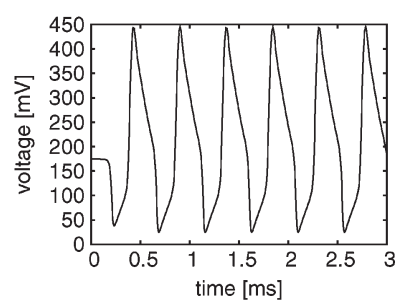

(b)
Fig. 9. VDP circuit and its behavior. (a) VDP circuit. (b) Simulation result.

is constructed by using an analog circuit instead of a digital circuit, which is composed of a large number of transistors.

In this section, we realize the proposed model and confirm that the synchrony, which reflects the behavior of the circuit, can be controlled by network transformation. We examine the behavior using a circuit simulator (LT Spice).

\section{A. VDP Circuit}

First, we develop a circuit composed of the previously mentioned VDP oscillators. The oscillation of a VDP oscillator originates from the nonlinearity of the tunnel diode, and the circuit can be assembled by a simple analog circuit of tunnel diodes, resistances, capacitors, and coils. We construct a VDP circuit on a circuit simulator, as shown in Fig. 9(a). We used the $1 \mathrm{~N} 3858$ tunnel diode, whose $V-I$ characteristics are shown in Fig. 8. The circuit model demonstrates an approximately $2-\mathrm{kHz}$ oscillation [as shown in Fig. 9(b)], with the following parameters: $R_{1}, R_{2}, C_{1}$, and $L_{1}=100 \Omega, 100 \Omega, 1 \mu \mathrm{F}$, and $500 \mathrm{mH}$, respectively. We use this VDP circuit as an oscillator.

\section{B. Antennal Lobe Circuit}

Next, the proposed behavior switch is constructed on the circuit simulator. A network is built with no local connections among the oscillators (Fig. 6), as shown in Fig. 10(a); the inductances of the oscillators $O_{1}$ to $O_{3}$ range from 300 to $700 \mathrm{mH}$, and they are connected with $400-\Omega$ resistances. Here, the resistance values correspond to the connection coefficients. As a result, the eigenfrequencies of the oscillators become approximately $2.5,2$, and $1.5 \mathrm{kHz}$, respectively. By providing a $2.3-\mathrm{kHz}$ sine oscillation as stimulation, which is the average frequency of $O_{1}$ and $O_{2}$, the synchronization of these two oscillators is achieved; only $\mathrm{O}_{3}$ oscillates independently [Fig. 10(b)]. In the same manner, by using an oscillation of approximately $1.7 \mathrm{kHz}$, $\mathrm{O}_{2}$ and $\mathrm{O}_{3}$ are synchronized.

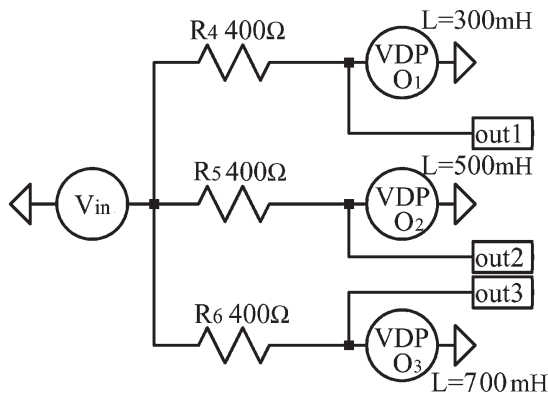

(a)

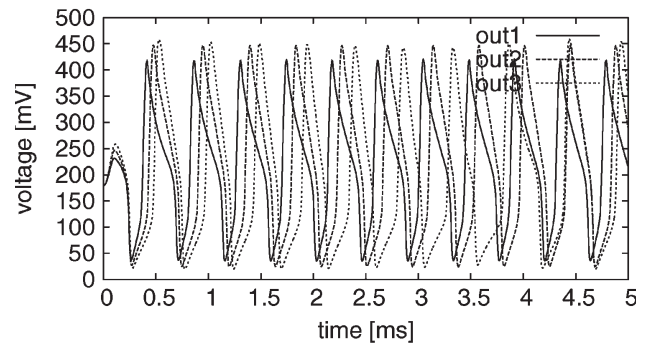

(b)

Fig. 10. Behavior of antennal lobe (AL) model (no LIN). (a) Model circuit. (b) Simulation result.

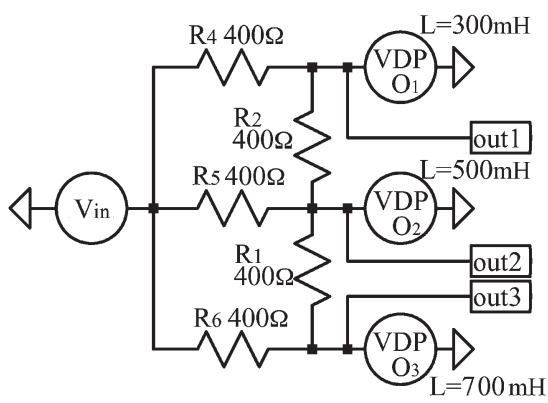

(a)

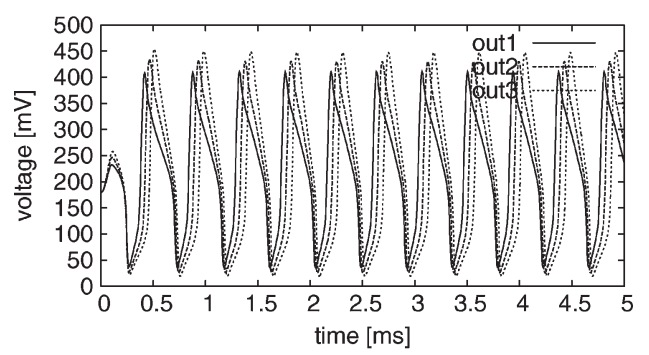

(b)

Fig. 11. Behavior of AL model (with LIN). (a) Model circuit. (b) Simulation result.

Subsequently, the local joints are taken into account. On the basis of the local connections of the proposed circuit (Fig. 7), additional pairs of oscillators are connected by using resistors, as shown in Fig. 11(a). Subsequently, the developed circuit is stimulated with the $2.3-\mathrm{kHz}$ input oscillation. Consequently, every oscillator is synchronized, as shown in Fig. 11(b). The same result is obtained for a $1.7-\mathrm{kHz}$ input.

Eventually, it is proved that a change in the local connection changes the response of the oscillator in the proposed model; therefore, we can state that the influence of NO, i.e., the influence of experience on the behavior of a cricket, is modeled. 


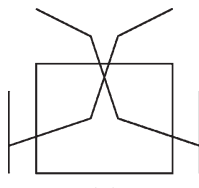

(a)

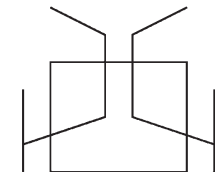

(b)
Fig. 12. Braitenberg vehicle. (a) Aggressive behavior. (b) Avoidance behavior.

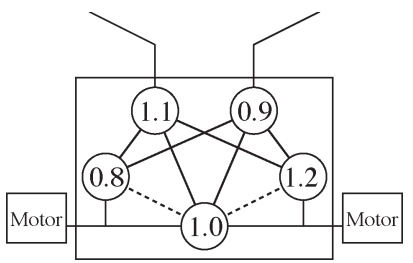

Fig. 13. Proposed robot model.

TABLE III

INFLUENCE OF SHORTCUT ON ROBOT BEHAVIOR

\begin{tabular}{|c|c|c|c|}
\hline State & Sensor input & Action & Behaviour \\
\hline no shortcuts & left input & left turn & aggressive \\
\cline { 2 - 4 } & right input & right turn & aggressive \\
\hline \multirow{2}{*}{ with shortcuts } & left input & forward movement & avoidance \\
\cline { 2 - 4 } & right input & forward movement & avoidance \\
\hline
\end{tabular}

\section{Robot Model With Aggressive AND AVOIDANCE BEHAVIORS}

\section{A. Robot Model}

In order to consider the proposed model from the viewpoint of behavior and to apply it to multiagent systems, we propose a robot model based on the aforementioned circuit. The simple robot models shown in Fig. 12 display both an offensive and cowardly behavior [22], [23]. If we cross-connect the motors and sensors such that the left sensor activates the right motor, as shown in Fig. 12(a), the robot heads toward the stimuli in a display of attacking behavior. On the other hand, if we arrange the sensors and motors in parallel [Fig. 12(b)], the robot attempts to avoid the source of the stimuli.

We apply the proposed oscillator circuit to this simple robot so that the aggressive and avoidance behaviors alternate, depending on the internal state. We arrange the proposed antennal lobe model between the sensor and the motor, as shown in Fig. 13. The circles in the figure indicate oscillators, and the numbers represent their eigenfrequencies. If there are no shortcuts (dotted lines), this robot moves in a direction that is toward the sensory input. On the contrary, if there are shortcuts, the frequencies of the oscillators change from 0.8 to 1.0 and 1.2 to 1.0 ; as a result, both the motors are activated by the stimuli. In this case, the robot attempts to leave the space where there is a sensor input; therefore, this behavior can be regarded as an avoiding action (Table III shows the complete reaction). Therefore, the proposed robot, like crickets, alternates its behavior by the transformation of its inner network.

\section{B. Realization}

On the basis of the aforementioned discussions, we construct a robot by using an analog circuit, as shown in Fig. 14. The circuit consists of five VDP oscillators. The oscillators are

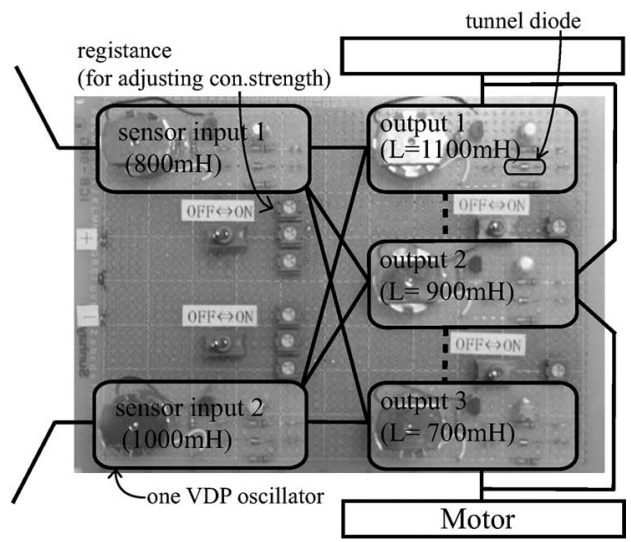

Fig. 14. Developed circuit.

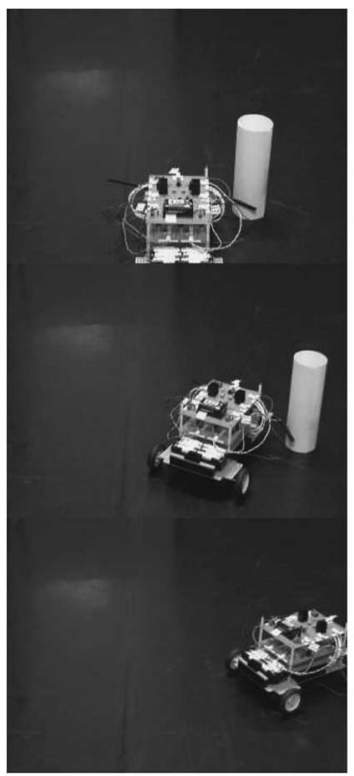

(a)

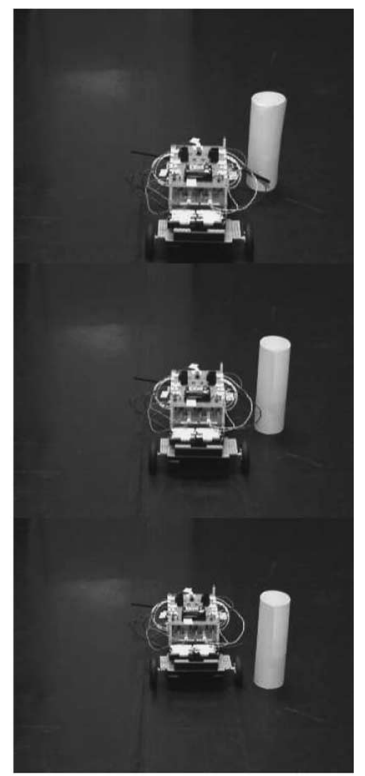

(b)
Fig. 15. Behavior of the circuit. (a) No shortcuts. (b) With shortcuts.

connected by resistances; the connections are shown by lines in Fig. 14. Changes in the structure are implemented by using the connections represented by the dotted lines: output 1output 2 and output 2-output 3. The two oscillators on the left-sensor inputs 1 and 2-are equipped with mechanical switches. Stimuli by physical contacts are sensed by the switches, which then initiate the oscillation of the oscillators (sensor inputs 1 and 2). The other oscillators connect to motors; the motors are actuated if the two oscillators that are directly connected to the motors are synchronized. The actuation is realized by placing a threshold on the signal between the output of the oscillator and the motor. Considering that the overlaid oscillation signals the most enhanced and the amplitude of the oscillation signals exceed the level of the above mentioned threshold when they are synchronized, motors are driven only at that time.

The robot's behavior is shown in Fig. 15. If there are no shortcuts (dotted lines in Fig. 13), the robot changes its orientation and goes toward the obstacle [Fig. 15(a)]. This behavior corresponds to the aggressive behavior of male crickets. However, if there are shortcuts (the $\mathrm{NN}$ is transformed after the fighting 
experience), it behaves as shown in Fig. 15(b). In this case, it does not go toward an obstacle but away from it. Thus, the robot displays cowardly tendencies due to the change in the network's structure. Thus, we have been able to manipulate the robot's behavior by changing the network's structure.

\section{Further Application}

The main characteristic of the proposed robot, i.e., the change in its behavior according to the physical connections, can be studied further if the circuit is made to be environmentally sensitive. For example, if we connect the oscillators to thermosensitive elements and arrange several robots in a field so that they start fighting due to their aggressive behavior, the offensive interaction between the robots generates heat, which results in a change in the connections. As a result, each robot will individually change its behavior to avoidance, and finally, a particular hierarchy will autonomously emerge among them.

\section{CONCLUSION}

In this paper, a method that uses the structural characteristics of a robotic device as a controller is proposed through the consideration of the change in the behavior of a cricket by neural network transformation. In this controller, the behaviors are distinguished by the synchrony of the oscillators based on biological evidences, and two olfactory functions, i.e., odor classification and behavior switching, are realized. Each component of the proposed network switch corresponds to a certain element in the brain. The oscillators correspond to neurons, and their connections correspond to synapses; therefore, this oscillator network is also applicable as an olfactory model.

We also confirm the attributes of the proposed model via a simple analog circuit composed of coupled VDP oscillators and develop a robot model that changes its action depending on physical network connections. The proposed robot demonstrates behavior selection on the basis of its physical mechanism and is, therefore, more sensitive to the environment than program-based robot models.

\section{REFERENCES}

[1] S. H. Strogatz, "Exploring complex networks," Nature, vol. 410, no. 6825, pp. 268-276, Mar. 2001.

[2] C. Y. Huang, C. T. Sun, J. L. Hsieh, and H. Lin, "Simulating SARS: Smallworld epidemiological modeling and public health policy assessments," J. Artif. Soc. Social Simul., vol. 7, no. 4, 2004.

[3] M. Small, P. Shi, and C. K. Tse, "Plausible models for propagation of the SARS virus," IEICE Trans. Fundam. Electron. Commun. Comput. Sci., vol. E87-A, no. 9, pp. 2379-2386, 2004.

[4] L. F. Lago-Fernandez, R. Huerta, F. Corbacho, and J. A. Siguenza, "Fast response and temporal coherent oscillations in small-world networks," Phys. Rev. Lett., vol. 84, no. 12, pp. 2758-2761, Mar. 2000.

[5] D. J. Watts, Small Worlds. Princeton, NJ: Princeton Univ. Press, 1999.

[6] H. Aonuma and K. Niwa, "Nitric oxide regulates the levels of cGMP accumulation in the cricket brain," Acta Biol. Hung., vol. 55, no. 1-4, pp. 65-70, May 2004.

[7] H. Aonuma, M. Iwasaki, and K. Niwa, "Role of no signaling in switching mechanisms in the nervous system of insect," in Proc. SICE Annu. Conf., Sapporo, Japan, Aug. 2004, vol. 3, pp. 2477-2482.

[8] T. Funato and D. Kurabayashi, "Network structure for control of coupled multiple non-linear oscillators," IEEE Trans. Syst., Man, Cybern. B, Cybern., vol. 38, no. 3, pp. 675-681, Jun. 2008.

[9] T. Funato, D. Kurabayashi, and M. Nara, "Synchronization control by structural modification of nonlinear oscillator network," in Proc. Distrib. Auton. Robot. Syst., Minneapolis, MN, Jul. 2006, pp. 41-50.

[10] M. Stopfer, M. Wehr, K. Macleod, and G. Laurent, "Neural dynamics, oscillatory synchronisation, and odour codes," in Insect Olfaction, B. S. Hansson, Ed. New York: Springer-Verlag, 1999, ch. 6, pp. 163-180.

[11] G. Laurent, M. Wehr, and H. Davidowitz, "Temporal representations of odors in an olfactory network," J. Neurosci., vol. 16, no. 12, pp. 3837-3847, Jun. 1996.

[12] S. A. Adamo and R. R. Hoy, "Agonistic behavior in male and female field crickets, Gryllus bimaculatus, and how behavioral context influences its expression," Anim. Behav., vol. 49, no. 6, pp. 1491-1501, Jun. 1995.

[13] P. A. Stevenson, H. A. Hofmann, K. Schoch, and K. Schildberger, "The fight and flight responses of crickets depleted of biogenic amines," J. Neurobiol., vol. 43, no. 2, pp. 107-120, May 2000.

[14] R. Okada, M. Sakura, and M. Mizunami, "Distribution of dendrites of descending neurons and its implications for the basic organization of the cockroach brain," J. Comp. Neurol., vol. 458, no. 2, pp. 158-174, Mar. 2003.

[15] V. E. D'yakonova and A. L. Krushinskii, "Effects of an NO synthase inhibitor on aggressive and sexual behavior in male crickets," Neurosci. Behav. Physiol., vol. 36, no. 5, pp. 565-571, Jun. 2006.

[16] F. C. Hoppensteadt and E. M. Izhikevich, "Oscillatory neurocomputers with dynamic connectivity," Phys. Rev. Lett., vol. 82, no. 14, pp. 2383-2386, Apr. 1999.

[17] Z. Li, "A neural model of contour integration in the primary visual cortex," Neural Comput., vol. 10, no. 4, pp. 903-940, May 1998.

[18] M. Barahona and L. M. Pecora, "Synchronization in small-world systems," Phys. Rev. Lett., vol. 89, no. 5, p. 054 101, Jul. 2002.

[19] A. Jadbabaie, N. Motee, and M. Barahona, "On the stability of the Kuramoto model of coupled nonlinear oscillators," in Proc. Amer. Control Conf., Boston, MA, Jun. 2004, pp. 4296-4301.

[20] L. M. Pecora and T. L. Carroll, "Master stability functions for synchronized coupled systems," Phys. Rev. Lett., vol. 80, no. 10, pp. 2109-2112, Mar. 1998.

[21] A. Stefanski, P. Perlikowski, and T. Kapitaniak, "Ragged synchronizability of coupled oscillators," Phys. Rev. E, Stat. Phys. Plasmas Fluids Relat. Interdiscip. Top., vol. 75, no. 1, p. 016210, Jan. 2007.

[22] V. Braitenberg, Vehicles: Experiments in Synthetic Psychology. Cambridge, MA: MIT Press, 1984.

[23] R. Pfeifer and C. Scheier, Understanding Intelligence. Cambridge, MA: MIT Press, 1999.

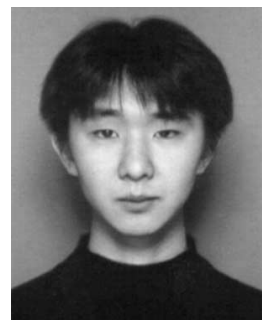

Tetsuro Funato (S'07) received the B.E. and M.E. degrees from the Tokyo Institute of Technology, Tokyo, Japan, in 2003 and 2005, respectively, where he is currently working toward the Ph.D. degree in the Department of Mechanical and Control Engineering.

His research interest includes group intelligence and biorobotics.

Mr. Funato is a member of The Robotics Society of Japan and the Society of Instrument and Control Engineers.

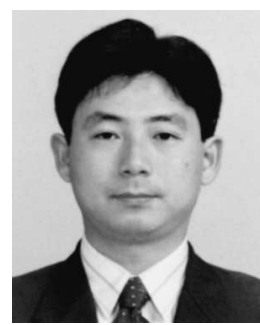

work, and biorobotics.

Dr. Kurabayashi is a member of The Robotics Society of Japan, Japan Society of Mechanical Engineers, Japan Society for Precision Engineering, and the Society of Instrument and Control Engineers. 


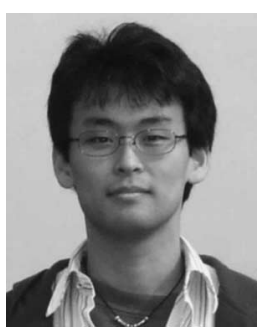

Masahito Nara received the B.E. degree from the Tokyo Institute of Technology, Tokyo, Japan, in 2004, where he is currently working toward the M.E. degree in the Department of Mechanical and Control Engineering.

His research interests includes distributed autonomous systems and group intelligence.

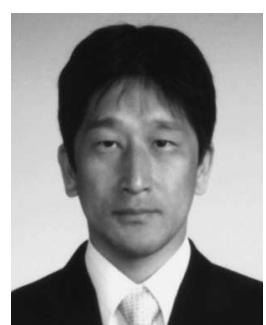

Hitoshi Aonuma received the B.S., M.S., and D.Sc. degrees from Hokkaido University, Sapporo, Japan, in 1991, 1993, and 1998, respectively.

From 1995 to 1996 , he was with the Graduate School of Science, Hokkaido University, as a Japan Society for the Promotion of Science (JSPS) Research Fellow. In 1998, he was a Research Associate for the Biotechnology and Biological Sciences Research Council, School of Biological Sciences, University of Southampton, Southampton, U.K., where he was a JSPS Research Fellow from 1999 to 2000 From 2001 to 2003, he was an Assistant Professor with the Research Institute for Electronic Science, Hokkaido University, where he has been an Associate Professor since 2003. His research interests include neuroethology, neurobiology, and animal physiology.

Dr. Aonuma is a member of the International Society for Neuroethology, the International Society for Invertebrate Neurobiology, Zoological Society of Japan, Japanese Society for Comparative Physiology and Biochemistry, and the Society of Evolutionary Studies. 